\title{
Design and Realization of Greenhouse Sensor Intelligent Management System Based on Internet of Things
}

\author{
https://doi.org/10.3991/ijoe.v13i05.7051 \\ Juanjuan Li \\ Shaanxi Institute Of International Trade \& Commerce, Shaanxi, China \\ vscrel914275@126.com
}

\begin{abstract}
Traditional and inefficient agricultural production methods cannot meet the modern agriculture requirements of safe, high quality, efficiency and productivity. The technology of Internet of Things is introduced into the field of agriculture, and the agricultural industrialization and information technology has an unprecedented opportunity. The relevant literature is read, the actual scene is investigated and the needs of agricultural field monitoring are identified. In the meanwhile, the development trend of Internet of Things and facility agricultural monitoring system is analyzed and system performance indicators that meet the actual requirements are developed. Moreover, the overall program of the system is designed and the three-tier architecture of Internet of Things system based on sensor technology, wireless communication technology, and configuration monitoring technology is constructed. The structure of the three layers of the sensing layer, transmission layer and application layer is analyzed, and the greenhouse sensor intelligent management system based on Internet of Things is designed. The performance of the system is tested in the laboratory. The test items include remote monitoring effect, information acquisition precision and system overall coordination. The results showed that the system is reasonable, the structure is compact, the network layer is reliable, and the performance is stable. Meanwhile, the application layer is rich in functionality, the interface is beautiful, the data processing is intelligent and the operability is strong. As last, it is concluded that the system meets system design requirements and expected performance specifications.
\end{abstract}

Keywords-Internet of Things, greenhouse sensor, wireless sensor, intelligent management system

\section{Introduction}

Facility agriculture is the general term for the automation, digitization and intelligent equipment system that serves agricultural production. The facility agriculture based on the Internet of things makes full use of sensor technology, wireless communication technology and information fusion processing technology, so as to construct intelligent management of agricultural production [1]. Therefore, the transition from traditional agriculture to intelligent agriculture will be accelerated, and the crop quality will be improved. 
The method is proposed for low degree automation, poor monitoring and supervision, serious waste of human and material resources and other issues of farmland, fruit trees and other large areas of outdoor planting area. In this paper, we study the remote intelligent monitoring of facility agriculture based on the Internet of Things on the basis of technical requirements and user needs [2]. We also research the facility agriculture remote intelligent information monitoring system based on Internet of Things, which can be used for farmland, fruit trees and other open-air large-scale agricultural production base, so as to achieve a comprehensive coverage of the agricultural planting site wireless sensor network $[4,5]$. Therefore, the important environmental parameters will be acquired digitally, and the information will be sent reliably based on wireless remote technology. And the intelligent application and analysis of the monitoring center will be realized, so as to improve the crop quality and production efficiency.

\section{State of the art}

As early as 1960s, foreign countries have begun the greenhouse research and application. At present, in the agricultural intelligence and environmental information collection, they have done very precise. For example, through satellite technology combined with weather conditions, France has achieved the forecast of pests and diseases in the greenhouse. As a result, they have increased crop productivity. Intelligent aquaculture management system has been widely applied to Europe and the United States. The automation management of the aquaculture industry can provide automatic water supply, collecting data and automatic alarm function. Compared with foreign countries, the domestic intelligent greenhouse research started late. However, with the development of science and technology, the Internet of things technology has been applied in the field of agricultural information in china. Liu Minghui et al. applied Web and GIS to study the expert system for prediction of agricultural pests and diseases. Chinese scholars use GSM short message technology to achieve real-time control of the greenhouse. By using sensor technology, they collected real-time environmental information such as air temperature and humidity, soil temperature and humidity, carbon dioxide concentration, soil $\mathrm{pH}$ and light intensity in the target monitoring area, so as to achieve the ultimate goal of improving crop yields. According to reports, the use of RFID technology can detect fruit trees, and analyze the growth of fruit. It can also realize the real-time monitoring of soil moisture, temperature and so on, so as to provide the basis for the study of the subsequent plant growth. In Xinjiang, China's researchers established a drip irrigation control system. It can be used to automatically monitor soil moisture information for crop growth. In accordance with the soil moisture for automatic drip irrigation, it can achieve the purpose of saving agricultural water. Thus it can be seen that our scientists have realized the intelligent control of the greenhouse. However, due to the high cost, it is difficult to implement on a large scale.

In summary, although the traditional intelligent greenhouse system can meet the basic requirements, it has many disadvantages, such as expensive equipment, complex 
internal protocol and high power consumption. There are still some problems in the practical application of many products. Therefore, the relevant literature is read, the actual scene is investigated and the needs of agricultural field monitoring are identified. In the meanwhile, the development trend of Internet of Things and facility agricultural monitoring system is analyzed and system performance indicators that meet the actual requirements are developed. Moreover, the overall program of the system is designed and the three-tier architecture of Internet of Things system based on sensor technology, wireless communication technology, and configuration monitoring technology is constructed. The structure of the three layers of the sensing layer, transmission layer and application layer is analyzed, and the greenhouse sensor intelligent management system based on Internet of Things is designed. At last, the performance of the system is tested in the laboratory. The test items include remote monitoring effect, information acquisition precision and system overall coordination.

\section{Overall Design of Greenhouse Sensor Intelligent Management System Based on Internet of Things}

\subsection{System design requirements analysis}

China's agricultural planting area is vast, and the main management mode in our country is individual farmer management, so the control intensity is low. In order to achieve the goal of intelligent monitoring of agricultural planting, the system design needs are analyzed as follows:

(1) We need to choose the modular form in system, so when a single module has problem, it will not have an impact on the remaining modules, and also will not cause the system to crash. At the same time, we can add or delete the components as needed.

(2) Multi-client access mechanism should be considered in system design, and it should be able to connect INTERNET, mobile phones and other terminal with network form.

(3) System should contain effective operational authority, namely, authorized users can flexibly use it, and the unauthorized users cannot access it.

(4) Unified data exchange platform should be built to meet the demand of node data exchange of unified deployment and distribution deployment. This system should conform to the technical data of the unified data exchange platform to realize the data exchange with other applications.

\subsection{System overall program design}

Greenhouse sensor intelligent management system based on Internet of Things contains several parts as follows. It combines the sensor, wireless communication, information fusion and processing and other key technologies collect the necessary parameters for crop growth [3,6-8], so as to complete parameter fusion and data mining. Remote monitoring and intelligent management is realized by hardware and 
software platform of Internet and Things $[9,11]$. The system is mainly composed of sensing layer, network layer and application layer. The overall system architecture is shown in Figure 1.

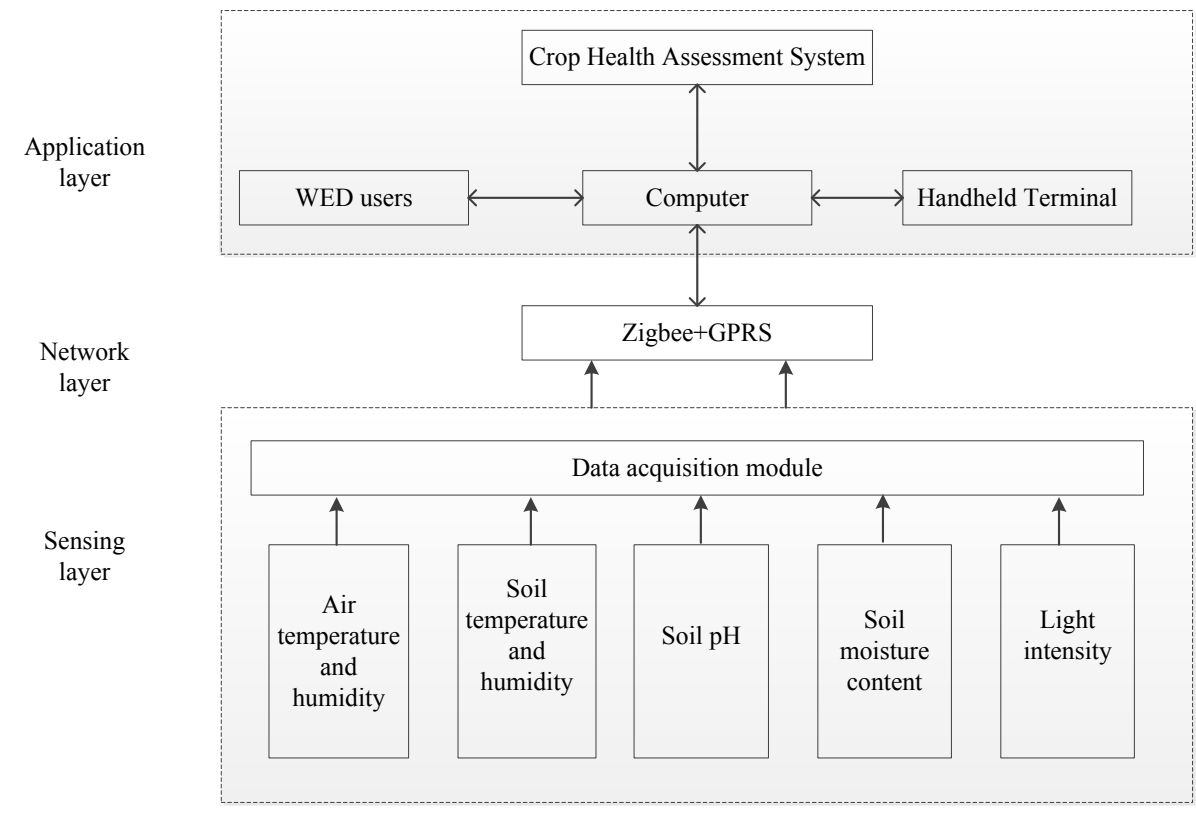

Fig. 1. Overall system architecture

(1) Sensing layer

The sensing layer is the bottom and the base layer of the facility agricultural networking. It mainly used to obtain real-time environmental monitoring of crop growth. The sensing layer mainly collects important environmental parameters such as soil temperature and humidity, air temperature and humidity, light intensity and soil PH.

(2) Network layer

The network layer is a system channel based on the local area network, wide area network and Internet, its main responsibility is data transmission, and it also can manage overall network system.

(3) Application layer

The application layer is located in the top of the system, is the system "nerve center" of decision-making. It is responsible for data display and storage, depth mining and utilization.

\subsection{System performance index}

This system is a complex system which integrates fine comprehensive collection, reliable and efficient transmission and intelligent application monitoring. It is based on the three-layer architecture of the Internet of Things to realize the comprehensive 
information sensing, the data transmission network, the intelligent management decision, the agricultural monitoring system [10]. This system improves monitoring and management efficiency of farmland, fruit forest, improves crop productivity, achieves effective regulation of crops and fruit trees, and proceeds remote real-time management of agricultural field crop growth status so as to achieve high precision, high reliability, and high sensitivity. The expected performance index as follows.

1. Rated voltage frequency: $220 \mathrm{VAC}, 50 \mathrm{~Hz}$.

2. Temperature: measuring range is $-30^{\circ} \mathrm{C}$ to $70^{\circ} \mathrm{C}$, measuring temperature accuracy is $\pm 0.2^{\circ} \mathrm{C}$.

3. Humidity: The measurement range is $(0-100) \% \mathrm{RH}$, and the measurement accuracy is $\pm 3 \% \mathrm{RH}$.

4. Light: measuring range is $0-200 \mathrm{klux}$, accuracy is $\pm 3 \%$.

5. Soil $\mathrm{PH}$ value: measurement range is $0-14$.

6. Zigbee outdoor communication distance: $30 \sim 1000 \mathrm{~m}$.

7. Zigbee sensitivity: $-97 \sim-103 \mathrm{dBm}$.

8. Zigbee number of channels: 16 .

9. Maximum serial data: 300Bytes.

10. Serial port rate: $110 \sim 230400 \mathrm{bits} / \mathrm{s}$.

11. GPRS transmit power: $<30 \mathrm{dBm}$.

12. Working environment requirements: temperature range is $-40^{\circ} \mathrm{C} \sim 85^{\circ} \mathrm{C}$, relative humidity range is $(0-100) \% \mathrm{RH}$.

\section{Design of Greenhouse Sensor Intelligent Management System Based on Internet of Things}

\subsection{System Sensing layer design}

The sensing layer is mainly used to obtain environmental microclimate monitoring information such as farmland, fruit trees and other agricultural planting sites [12]. The sensing layer is composed of a variety of sensors and signal conditioning circuits which detect different environmental microclimatic parameters, and realizes the realtime acquisition of agricultural field analog signals and environmental parameters. The acquisition of analog signals is obtained by ADC conversion circuit. The analog acquisition module transmits the collected sensor information to the Zigbee wireless module through the RS485 serial port, thus completing the information collection of the sensing layer.

1. Sensor design

(1) Air temperature sensor, humidity sensor

At present, the air temperature sensor and air humidity sensor is usually constructed in integrated processing unit, so it can measure temperature and humidity so as to reduce the number of sensors. The temperature can be measured by contact or noncontact. 
This system uses FM-KWS outdoor temperature and humidity sensors, the main technical parameters are shown in Table 1.

Table 1. Temperature and humidity sensors

\begin{tabular}{|c|c|}
\hline Parameters & Value \\
\hline Air humidity & $0-100 \%$ \\
\hline Air temperature & $-30-70{ }^{\circ} \mathrm{C}$ \\
\hline Signal output mode & $4-20 \mathrm{~mA}$ \\
\hline Operating voltage & $12-24 \mathrm{VDC}$ \\
\hline Response time & $<1 \mathrm{~S}$ \\
\hline Settling time & $1 \mathrm{~S}$ \\
\hline Operating temperature range & $-35 \sim 75^{\circ} \mathrm{C}$ \\
\hline
\end{tabular}

(2) Soil temperature and humidity sensor

Soil temperature and humidity are generally measured by plug-in temperature and humidity sensor. Plug-in temperature and humidity sensor is relatively small, it has multiple probes and the length of each probe is different.

This system uses ZY-ES212 soil temperature and humidity sensors, the main technical parameters are shown in Table 2.

Table 2. Soil temperature and humidity sensor

\begin{tabular}{|c|c|}
\hline Parameters & Value \\
\hline Soil temperature & $-30-70{ }^{\circ} \mathrm{C}$ \\
\hline Soil moisture & $0-100 \%$ \\
\hline Signal output mode & $4-20 \mathrm{~mA}$ \\
\hline Operating Voltage & $12-24 \mathrm{VDC}$ \\
\hline Response time & $<1 \mathrm{~S}$ \\
\hline Measuring stability time & $1 \mathrm{~S}$ \\
\hline Operating temperature range & $-30-70^{\circ} \mathrm{C}$ \\
\hline Soil moisture measurement area & $\begin{array}{c}\text { A cylinder with a diameter of } 3 \mathrm{~cm} \text { and a height of } 7 \mathrm{~cm} \\
\text { centered on central probes }\end{array}$ \\
\hline
\end{tabular}

(3) Soil $\mathrm{pH}$ sensor

Soil $\mathrm{pH}$ is the index of soil acidity and alkalinity, which affects the fertility and nature of soil. $\mathrm{PH}$ value affects the synthesis and decomposition of important mineral organic elements in the soil, so it is an important factor affecting the health of crops. Soil $\mathrm{pH}$ measurements include colorimetric and potentiometric methods.

This system uses PH-TRSJ soil $\mathrm{pH}$ sensor, the main technical parameters are shown in Table 3.

Table 3. Soil pH sensor

\begin{tabular}{|c|c|}
\hline Parameters & Value \\
\hline Measuring range & $0-14 \mathrm{PH}$ \\
\hline Signal output mode & $4-20 \mathrm{~mA}$ \\
\hline Operating Voltage & $12-24 \mathrm{VDC}$ \\
\hline Response time & $<10 \mathrm{~S}$ (in water) \\
\hline Operating temperature range & $0-80^{\circ} \mathrm{C}$ \\
\hline Operating humidity range & $0-95 \%$ \\
\hline
\end{tabular}


(4) Soil salinity sensor

The measurement principle of soil salinity sensor is to indirectly insert the probe into the measured object, and the impedance is converted to the corresponding linear voltage signal for the post-circuit processing. The voltage value is inversely proportional to the impedance of measured object, proportional to the conductance value, and is proportional to the soil solution concentration.

This system uses the TM-EC soil salt sensor, and the main technical parameters are shown in Table 4.

Table 4. Soil salinity sensor

\begin{tabular}{|c|c|}
\hline Parameters & Value \\
\hline Salt range & $0-5 \mathrm{mS} / \mathrm{cm}$ \\
\hline Resolution & $0.1 \mathrm{mS} / \mathrm{cm}$ \\
\hline Output signal & $4-20 \mathrm{~mA}$ \\
\hline Operating Voltage & $12-24 \mathrm{VDC}$ \\
\hline
\end{tabular}

(5) Light intensity sensor

Light intensity is the measurement mode of brightness. The measurement method of light intensity is divided into thermoelectric effect measurement and photoelectric conversion effect measurement. This system uses the model NA2D10 light intensity sensor, and the main technical parameters are shown in Table 5.

Table 5. Light intensity sensor

\begin{tabular}{|c|c|}
\hline Parameters & Value \\
\hline Range & $0-2000001 \mathrm{x}$ \\
\hline Output signal & $4-20 \mathrm{~mA}$ \\
\hline Operating Voltage & $12-24 \mathrm{VDC}$ \\
\hline Operating temperature range & $-40-80^{\circ} \mathrm{C}$ \\
\hline Operating humidity range & $0-100 \%$ \\
\hline
\end{tabular}

2. Analog acquisition module selection

The system uses RS-4021M analog acquisition module as the system I/O port expansion. The module has the following characteristics:

1. Analog input channel is the 16-channel single-ended or 8-channel differential;

2. Input range are $\pm 10 \mathrm{~V}, \pm 5 \mathrm{~V}, \pm 2.5 \mathrm{~V}, \pm 1.25 \mathrm{~V}, 0 \sim 20 \mathrm{~mA}$ (need an external precision resistance of $250 \Omega, 0.1 \%$ ), $4 \sim 20 \mathrm{~mA}$ (need an external precision resistance of $250 \Omega, 0.1 \%$ ).

3. Sampling frequency: 400 times /sec.

4. Communication protocol: ModbusRTU.

5. Communication interface: RS485 bus.

6. Resolution: 12-bit.

7. Accuracy: $\pm 0.1 \%$.

8. Isolation voltage: $2500 \mathrm{~V}$. 


\subsection{System network layer design}

The network layer is the "neural network channel" that transmits information to the brain. It determines whether the information collected by the front end can be transmitted effectively to the "brain" of the central information application monitoring center of the host computer.

1. Zigbee node design

(1) Zigbee module selection

The Zigbee node module is the basis for building a wireless sensor network. It is responsible for communicating with the gateway, forwarding the data of the information acquisition module to the Zigbee-GPRS gateway, or sending the gateway data command to all terminal nodes to monitor the crop growth environment.

According to the actual requirements, this system uses F8914 as Zigbee node, so as to achieve the scene and the gateway communication.

The technical parameters of the F8914Zigbee module are shown in Table 6.

Table 6. Technical parameters of the F8914Zigbee module

\begin{tabular}{|c|c|}
\hline Standard and frequency bands & IEEE802.15.4、ISM2.4Hz \\
\hline Indoor communication distance & $30-90 \mathrm{~m}$ \\
\hline Outdoor communication distance & $500-2000 \mathrm{~m}$ \\
\hline Transmit power & $2.82-100 \mathrm{~mW}$ \\
\hline Theoretical bandwidth & $250 \mathrm{Kbps}$ \\
\hline Sensitivity & $-97-103 \mathrm{dBm}$ \\
\hline Network topology & Point-to-point, point-to-multipoint or mesh \\
\hline Number of channels & 16 \\
\hline channel & 300 Bytes \\
\hline Maximum serial data & \\
\hline
\end{tabular}

(2) Zigbee address allocation strategy

Zigbee devices usually have two types of addresses. The first one is 64-bit IEEE long address, that is, MAC address, which is unique in the world. The other is 16-bit short address, which is to facilitate the programming in the actual communication applications, so as to mark them and identify each other, also known as the network address.

The types of Zigbee network address are shown in Table 7.

Table 7. Zigbee network address type

\begin{tabular}{|c|c|c|}
\hline Address type & Number of bits & Alternative name \\
\hline IEEE & $64-$ bit & MAC address \\
\hline & & Extended address \\
\hline Network & 16 -bit & Logical address \\
\hline & & Short address \\
\hline
\end{tabular}

(3) Zigbee network formation

The entire LAN network initiations, construction, join and departure is finished by programming with Zigbee primitives. The main program flow chart is shown in Figure 2. 


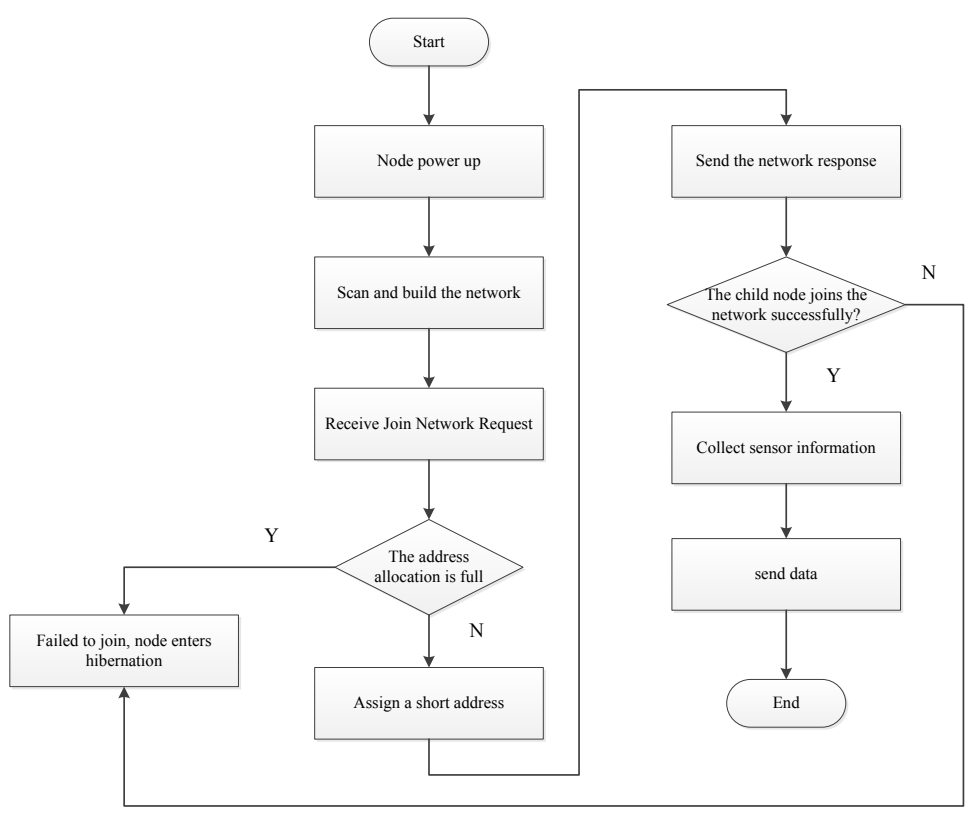

Fig. 2. Flow chart of new network building on Zigbee coordinator

After the installation, we can power the Zigbee device, the device's light is lit, so we can see that the device network is successful.

\section{GPRS Gateway design}

(1) GPRS module selection

GPRS digital transmission module is an essential component, which can extend the wireless sensor network distance. Communicate with the Zigbee of proximity LAN to get the microclimate information the bottom sensing layer, and complete the link with the Internet, so as to effectively transmit the agricultural field data effectively to the monitoring center.

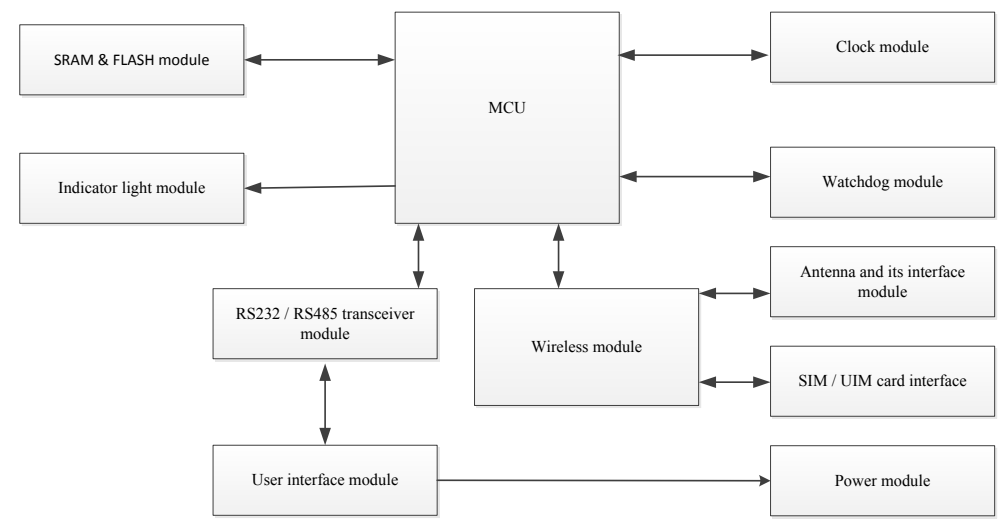

Fig. 3. Schematic diagram of gateway hardware structure 
Paper-Design and Realization of Greenhouse Sensor Intelligent Management System Based on Internet...

The technical parameters of F8114GPRS gateway module are shown in Table 8.

Table 8. F8114 module parameters

\begin{tabular}{|c|c|}
\hline Standard and frequency band & $\begin{array}{c}\text { EGS M900/GSM1800 dual-frequency } \\
\text { GPRS CLASS10 }\end{array}$ \\
\hline FLASH & $512 \mathrm{~KB}$ (Extensible to 8MB) \\
\hline SRAM & $256 \mathrm{~KB}$ (Extensible to 1MB) \\
\hline Transmit power & $<30 \mathrm{dBm}$ \\
\hline Theoretical bandwidth & $85.6 \mathrm{Kbps}$ \\
\hline Sensitivity & $<-107 \mathrm{Dbm}$ \\
\hline Serial rate & $110-230400 \mathrm{bits} / \mathrm{b}$ \\
\hline
\end{tabular}

As can be seen from the above, the module not only has GPRS cellular longdistance communication function, but also can interact with the Zigbee protocol as a gateway of Zigbee, GPRS, and Internet.

(2) GPRS network establishing

The mobile station (MS) provides GPRS users with the commonly used data connection status, including idle, standby, and ready. Each state has its own functional level and information status.

GPRS state division as shown in Table 9.

Table 9. GPRS state division

\begin{tabular}{|c|c|}
\hline State & Standard of division \\
\hline Idle & MS has not started or no GPRS attach \\
\hline Standby & $\begin{array}{c}\text { MS has completed GPRS connection, but no } \\
\text { data transmission }\end{array}$ \\
\hline Ready & Data transmit is in progress \\
\hline
\end{tabular}

GPRS has mobility, so the management of MS on GPRS has three states above. GPRS state transition includes five cases, respectively is idle to ready, wait to idle, wait to ready, ready to wait, and ready to idle.

After the setup is complete, we power on the device, and dial the F8114GPRSIP modem to test the performance. When the device is monitored by the central software, it means that F8114GPRSIP modem is successfully connected to the local IP address and the indicator light is powered on. So the data transmission of GPRS to Internet will be realized.

F8114 can be used as a coordinator for Zigbee, the module is embedded with Zigbee protocol. After the network is built, it will be networked with other Zigbee nodes. The data will be transmitted to GPRS by Zigbee convergence node based on F8114 "serial + network + Zigbee" program.

\subsection{System application layer design}

1. Information monitoring center hardware support platform selection

Application layer information monitoring center is the entire system server side. In the entire monitoring system, it has many functions such as communication, display, 
user login management, and data storage. The application layer PC of this system has a reliable server configuration. Combined with the installation requirements of the configuration software and agricultural field planting situation, the server configuration is as follows.

(1) Hardware environment

CPU: P4 processor, hardware: at least $80 \mathrm{G}$ and $1 \mathrm{GHz}$

(2) Operating system

Windows 2000 (SP4) / Windows XP（SP2）/Vista/Win7/Win8。

(3) Internet environment

The host has a public IP address.

2. Information Monitoring Center Software Design

Application layer information monitoring center needs to carry out remote monitoring of agricultural planting site. It can be seen from the figure that the function of the system can be divided into four parts, respectively is

Site monitoring: the situation of crop growth in the field of agricultural cultivation will be monitored in real time, current environmental information in farmland and fruit trees is shown in both figures and graphs in the way of image intuitive, clear information.

Automatic alarm: alarm function can be divided into two categories according to the principle of timeliness, respectively is real-time alarm information and historical alarm information

Curve display: Curve display in accordance with the timeliness can be divided into real-time curve display and historical curve display. We can visually observe the current trend of changes in monitoring by real-time curve display, extract effective historical information, and provide the basis for the adjustment of the planting plan and the improvement of the environment.

Report production: Report production is a significant feature of this system. It stores the on-site monitoring information in tabular form, examines the monitoring the real-time or historical information and summarizes the change regulation of the environmental monitoring.

3. Monitoring center interface design

(1) Variable definition of the monitoring center

The data types defined by the monitoring center are divided into two categories. The first one is I/O data, the second one is the memory variable for the interface itself.

The definition method of these two types of variables is similar with the interface. In general, when a memory variable performs data exchange, the intermediate variable is set to a memory variable.

The sensor signal rang selected by the system is $4 \sim 20 \mathrm{~mA}$, Zigbee module analog acquisition port input signal range is $0 \sim 20 \mathrm{~mA}$. The conversion formula for converting the analog quantity into the actual engineering value is shown in equation (1).

$$
Y=\frac{\left(X_{\max }-X_{\min }\right)(X-186.2)}{931-186.2}+X_{\min }
$$


$Y$ is actual data value of sensor, $X_{\max }$ is maximum value of sensor measurement range, $X_{\min }$ is minimum value of sensor measurement range, $X$ is the digital quantity converted by A/D conversion.

(2) System login interface

Login interface is "management portal" of the entire application layer. According to the system design requirements, application layer information monitoring center not only has a good human-computer interaction design, exquisite appearance, simple and powerful system menu, but also has enough security.

In accordance with the different user's management authority, the system divides the operator into different login levels. Each level has different user name and password, so as to prevent over-limit operation.

(3) System monitoring main interface

System monitoring main interface can reflect the overall monitoring effect of the system. It also can display sensory layer information through numbers and histograms. With the real-time refresh of the collection status, the situation of real planting site is reacted, so as to guide and analyze the growth status of the crop.

(4) Alarm interface

Alarm interface design is divided into real-time alarm and historic alarm. When the current acquisition value deviates greatly from the set range, real-time alarm system will alert the operator to take measures. The realization of the system's historic alarm is based on ACCESS database established. So we can query and analyze historical alarm data based on date and monitoring quantity.

(5) Data curve interface

The data curve can be divided into real-time curve and historical data curve. Curve is an important basis for analyzing the changing law of planting monitoring. It can clearly sum up the monitoring trends in the present or for a period of time.

(6) Report interface

According to the time period, the report can be divided into various forms. Among them, the monthly report and annual report are based on daily report. The daily report records the data according to the program and integrates the day monitoring quantity.

\section{$5 \quad$ System Test and Debugging}

Figure 4 is the greenhouse sensor intelligent system control model based on the Internet of things. 


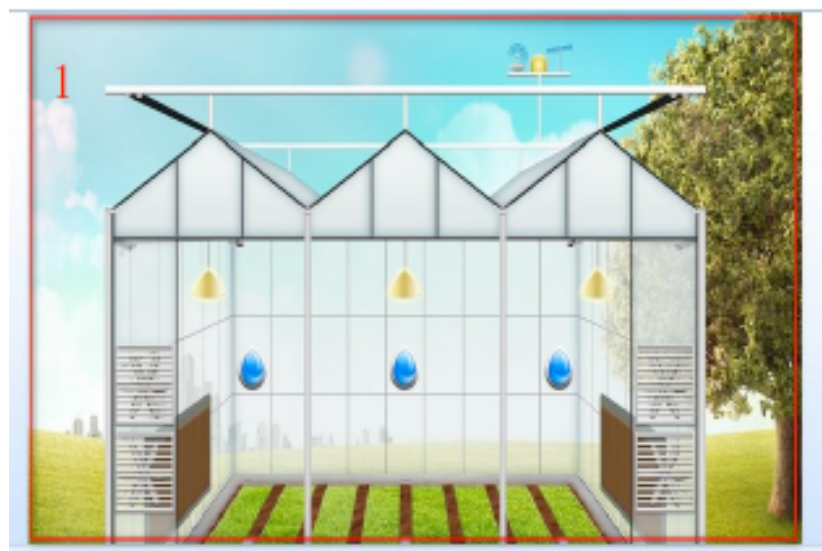

Fig. 4. Control model of greenhouse sensor intelligent system

Figure 5 is the greenhouse sensor intelligent system monitoring interface based on the Internet of things.

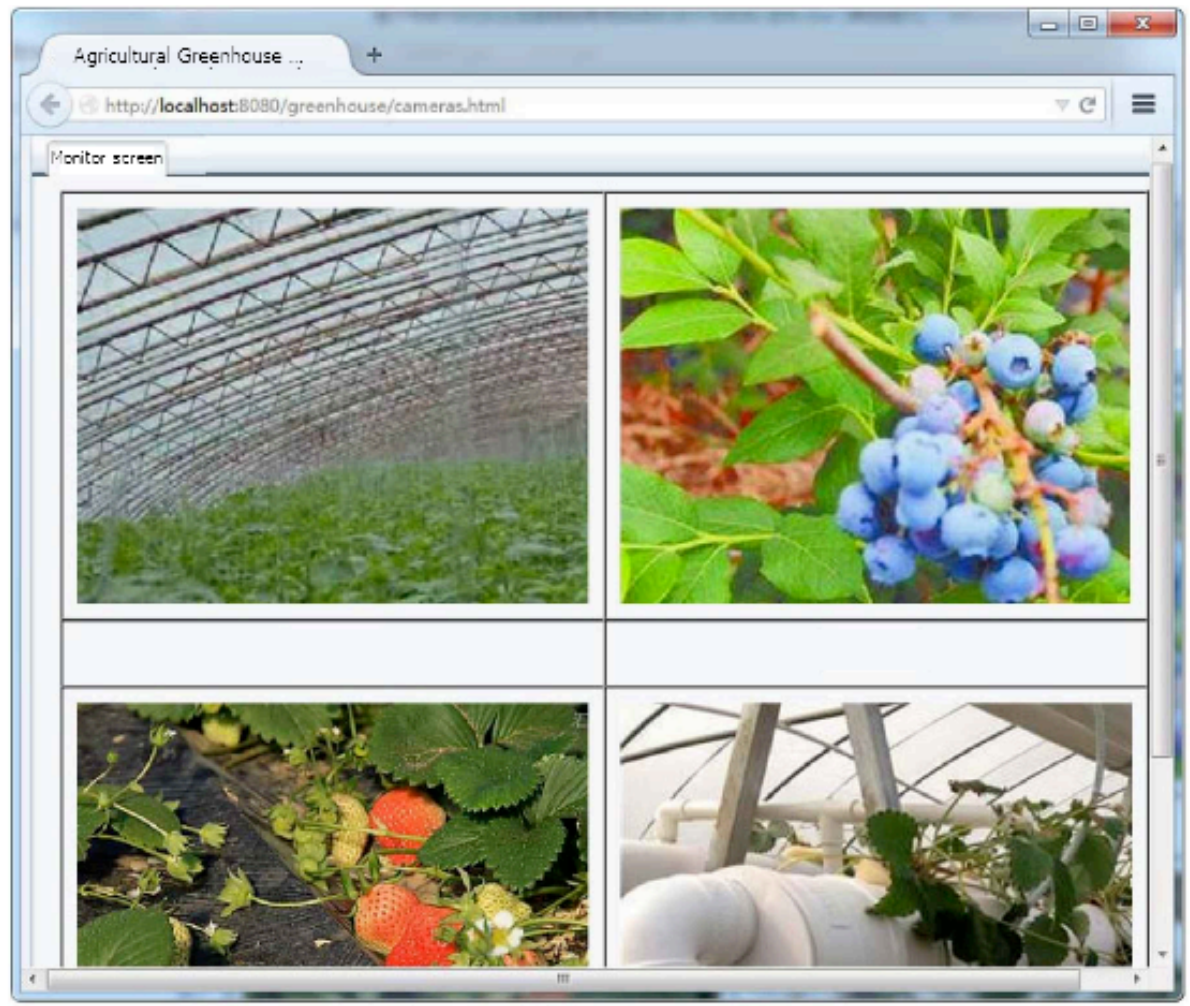

Fig. 5. Monitoring interface display 


\subsection{Remote monitoring effect test}

We mainly test the data transmission capability of GPRS and application layer monitoring center. So, the key part is whether the bottom data of the Zigbee proximity LAN can be connected to the application layer monitoring center.

System remote measurement humidity chart is shown in Figure 5.

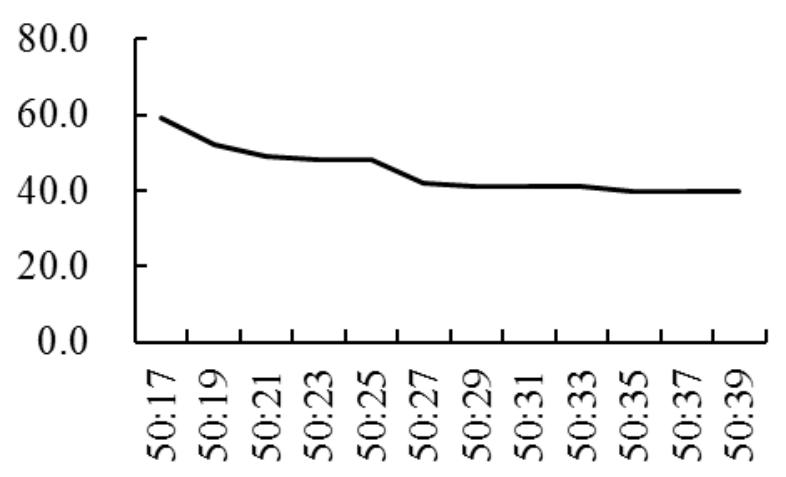

Fig. 6. System remote measurement humidity curve

As can be seen from Figure 5, the sensor data of sensing layer bottom can be effectively transmitted through the GPRS to the application layer monitoring center virtual serial port, and the data transfer rate is fast. In the data transfer process, the measured humidity curve has no break and the curve is smooth. So the remote transmission of wireless signals is reliable, so as to meet the system requirements

\subsection{Acquisition result accuracy test}

The experimental site is the laboratory of the fourth floor of the Central Mining Building. When the temperature and humidity sensor is accessed, we measure the temperature and humidity of laboratory. The indoor thermometer and hygrometer are used as standard values to calculate the sensor measurement accuracy.

In the process of experiment, we measured the temperature and humidity values for 10 times. The temperature data is shown in Figure 7, and the humidity data is shown in Figure 8. 


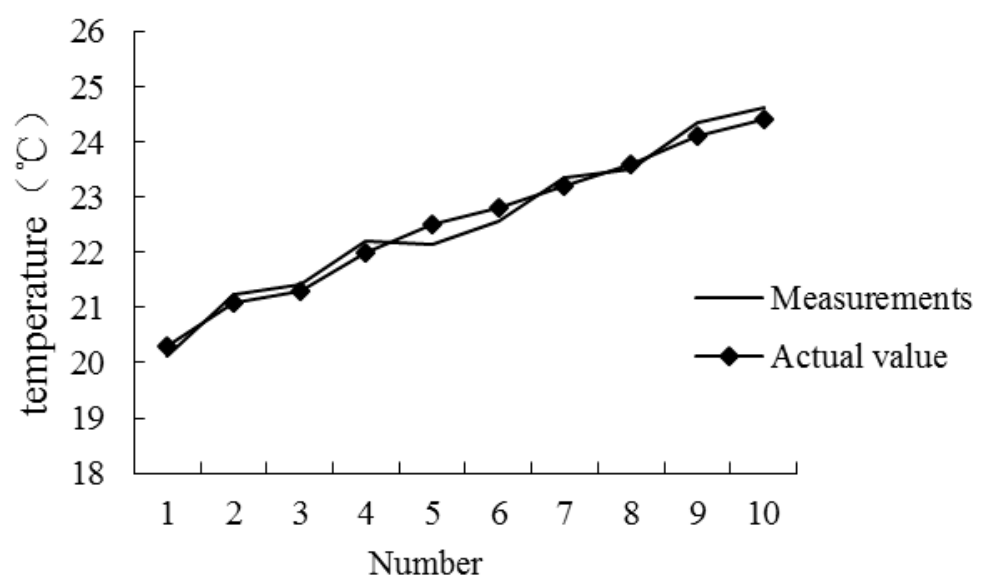

Fig. 7. Temperature test data

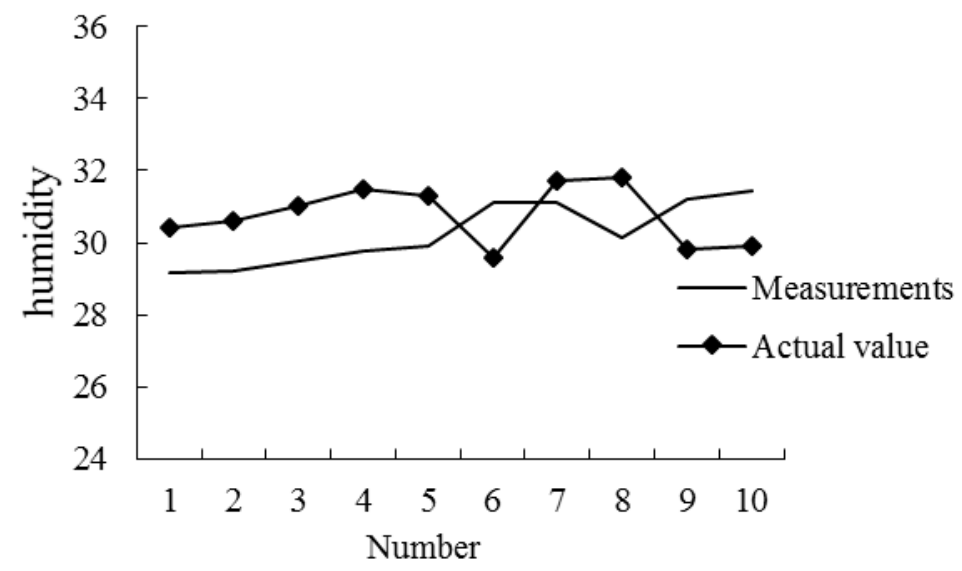

Fig. 8. Humidity test data

As can be seen from Figure 7 and Figure 8, the temperature average difference of collected value and the actual value is $0.194{ }^{\circ} \mathrm{C}$, the humidity average difference of collected value and the actual value is $1.503 \% \mathrm{RH}$. So it can meet the system performance and technical requirements and can be directly used for agricultural planting site.

\subsection{System integration and debugging}

System integration and debugging was completed under laboratory conditions. We simulated the node layout and installation environment of agricultural scene, integrated sensing layer, network layer, and application layer and proceeded the overall de- 
bugging. In the laboratory, the nodes are arranged on the bracket to form a wireless sensor network. We also debugged the data collection, data wireless LAN transmission, data wireless WAN transmission and data application layer display monitoring. The results show that the wireless communication performance of the system is good, monitoring data has high readability and the original design goal of the overall scheme was realized.

\section{Conclusions}

Based on the development situation of facility agriculture of Internet of things, this paper deeply analyzes the proximity LAN sensing layer structure of the wireless sensor network based on Zigbee nodes. We design the remote communication protocol based on GPRS, and develop the remote intelligent information monitoring system of greenhouse. Therefore, the intelligent management system of greenhouse sensor based on Internet of things is realized, and the remote intelligent information monitoring of the facilities networking is completed.

According to the system architecture and overall design scheme, we select the three-layer architecture model of the Internet of Things combined with the system performance index. Meanwhile, we design the sensing layer system and its core is sensor and analog acquisition module. We also determine the node unit of the wireless sensor network proximity LAN based on Zigbee and build a Zigbee and longdistance GPRS communication protocol heterogeneous fusion network. We achieve the communication of agricultural field and remote monitoring center, thereby breaking the geographical restrictions. We also complete the Zigbee network and GPRS network networking design on the basis of modular programming method and test it in the laboratory. This paper designs the program of the remote monitoring system, and analyzes GPRS and application layer virtual serial data transmission and reception situation, so as to achieve remote data collection and reliable transmission. By comparing the measured values with the theoretical values of temperature and humidity sensors, we can see that the error ranges achieve the accuracy goal of system data acquisition. According to the overall operation of the system, the system is integrated and debugged to optimize the network parameter. The robustness of the wireless sensor network is gradually increased. This method effectively improves the reliability of the system, and achieves the desired goal.

\section{$7 \quad$ References}

[1] Chen, C., Duan, S., Cai, T., Liu, B., \& Hu, G. (2011). Smart energy management system for optimal microgrid economic operation. IET renewable power generation, 5(3), 258267. https://doi.org/10.1049/iet-rpg.2010.0052

[2] Feng, C., Wu, H. R., Zhu, H. J., \& Sun, X. (2012). The design and realization of apple orchard intelligent monitoring system based on internet of things technology. Advanced Materials Research, 546-547, 898-902. https://doi.org/10.4028/www.scientific.net/AMR.546$\underline{547.898}$ 
Paper-Design and Realization of Greenhouse Sensor Intelligent Management System Based on Internet...

[3] Guo, T., \& Zhong, W. (2015). Design and implementation of the span greenhouse agriculture Internet of Things system. International Conference on Fluid Power and Mechatronics (pp.398-401). IEEE.

[4] Kyriakarakos, G., Piromalis, D. D., Dounis, A. I., Arvanitis, K. G., \& Papadakis, G. (2013). Intelligent demand side energy management system for autonomous polygeneration microgrids. Applied Energy, 103, 39-51. https://doi.org/10.1016/j.apenergy. 2012.10.011

[5] Milanes, V., Villagra, J., Godoy, J., Simo, J., Pérez, J., \& Onieva, E. (2012). An intelligent V2I-based traffic management system. IEEE Transactions on Intelligent Transportation Systems, 13(1), 49-58. https://doi.org/10.1109/TITS.2011.2178839

[6] Ni, R. (2014). Design and implementation of intelligent greenhouses based on the internet of things. Applied Mechanics \& Materials, 631-632, 188-191. https://doi.org/10.4028/ www.scientific.net/AMM.631-632.188

[7] Nissimov, S., Goldberger, J., \& Alchanatis, V. (2015). Obstacle detection in a greenhouse environment using the Kinect sensor. Computers and Electronics in Agriculture, 113, 104115. https://doi.org/10.1016/j.compag.2015.02.001

[8] Prabhu, S. B., Dhasharathi, C. V., Prabhakaran, R., Kumar, M. R., Feroze, S. W., \& Sophia, S. (2014). Environmental monitoring and greenhouse control by distributed sensor Network. International Journal of Advanced Networking and Applications, 5(5), 2060.

[9] Swan, M. (2012). Sensor mania! the internet of things, wearable computing, objective metrics, and the quantified self 2.0. Journal of Sensor and Actuator Networks, 1(3), 217-253. https://doi.org/10.3390/jsan1030217

[10] WANG, F., FANG, J., \& ZHANG, X. (2011). Research of environmental monitoring system for greenhouse based on wireless sensor network [J]. Journal of Northeast Agricultural University, 2, 015.

[11] Xia, F., Yang, L. T., Wang, L., \& Vinel, A. (2012). Internet of things. International Journal of Communication Systems, 25(9), 1101. https://doi.org/10.1002/dac.2417

[12] Xiong, Y., Cheng, H., Shen, M., He, W., Liu, Y., Zhao, L., . \& Liu, L. (2012). Design of intelligent greenhouse information management system with hybrid architecture. Transactions of the Chinese Society of Agricultural Engineering, 28(1), 181-185.

\section{Author}

Juanjuan Li is Associate Professor at Shaanxi Institute of International Trade \& Commerce, Shaanxi, China (vscre1914275@126.com).

Article submitted 07 April 2017. Published as resubmitted by the author 15 May 2017. 